\title{
Histopathology in Bovine Rotavirus (Type A) Infected Calves and Its Confirmation by ELISA and RT-PCR
}

\author{
Rakib TM, Barua SR*, Siddiki AMAMZ, Masuduzzaman M, Hossain MA and Chowdhury S \\ Department of Pathology and Parasitology, Chittagong Veterinary and Animal Sciences University, Bangladesh
}

Submission: May 25, 2018; Published: July 10, 2018

*Corresponding author: Barua SR, Department of Pathology and Parasitology, Chittagong Veterinary and Animal Sciences University, Khulshi, Chittagong 4225, Bangladesh, Email: samardvm27@gmail.com

\begin{abstract}
The present study was designed to investigate the pathology of bovine rotavirus A infection in dead calves with the history of diarrhea. The cases were further confirmed by ELISA and RT-PCR. A total of 7 dead calves were examined by recommended post mortem techniques followed by confirmatory diagnostic tools. Four calves were found positive by both ELISA and RT-PCR assays. Grossly, no characteristic pathological lesions were observed except congestion in small intestine. The characteristic histopathological lesions were observed mainly in jejunum, ileum, Peyer's patches and mesenteric lymphnode. Blunting of villi, lymphocytic infiltration, intracytoplasmic eosinophilic inclusion body, nuclear degradation and syncytia formation were observed under the microscope with different magnification. However, observation of pathological changes is important for identification rotaviral infection in case of doubtful specimens. To our knowledge, this is the first report from Bangladesh applying post-mortem examination, ELISA and RT-PCR for detection of bovine rotavirus group A from diarrheic dead calves.
\end{abstract}

Keywords: Pathology; ELISA; RT-PCR; Rotavirus; Dead calves; Jejunum; Ileum; Syndrome; Morbidity; Mortality; Nervous system; Population; Lymphocytes; Bouin's solution; Citric acid; Gelatin; Alcohol; Xyline mixture; Ultraviolet light; Etiology; Temperature

\section{Introduction}

Neonatal calf mortality is one of the most common animal health concerns for dairy industry which affect the herd health, farm profitability and as a whole the economy of the country. It has also been recognized as an important condition responsible for high morbidity and mortality in calf [1], particularly in the first month of age that was accounted about $84 \%$ of the total mortality [2,3].

Rotavirus represents as a significant cause of neonatal calf mortality which belongs to the family Reoviridae, genus Rotavirus and is non-enveloped, double stranded RNA virus, with a diameter of $65-70 \mathrm{~nm}$. Rotavirus infects primarily mature non-proliferating enterocytes lining the villi of small intestine. Rotavirus diarrhoea is caused by a combination of factors, which include reduction in epithelial surface area resulting in malabsorpation syndrome, activation of the enteric nervous system, and the effect of the rotavirus nonstructural protein [4]. Mild catarrhal changes in the small intestine with congestion of both mucosa and serosal layers are also found $[5,6]$. The severity and localization of rotavirus intestinal infection vary among animal species and between studies [7]; however, the pathological changes are almost exclusively limited to the small intestine. The small intestinal mucosa of neonatal calf consists of long finger-like villi, when challenged with different agents, the pathological changes are almost similar. The main pathological lesion consists of stunting and thickening of the villi. Frequently the villi are fused and leading to a flat mucosa in the severe cases. The epithelium covering such villi is generally cuboidal, and in rotavirus infection squamous epithelium covering the tip of some villi may be seen [3].

The presence of intracytoplasmic inclusion bodies in small intestinal enterocytes is the indication of viral etiology. The characteristic changes in rota viral infection are blunting of villi, thickening of crypts etc. The thinning of mesenteric lymph nodes is suggestive of lymphocytic migration as seen in the form of infiltration in mucosa. The histological changes in ileum are desquamation and necrosis of crypt with villous atrophy, infiltration of lymphocytes in inter villous areas and decreased population of lymphocytes in Peyer's patches and mesenteric lymph node [1,2]. The presence of rotavirus antigen frequently found in the intestinal tissues and mainly confined 
to the small intestinal mucosa. Infected cells stained with H\&E staining method showed characteristic syncytia and eosinophilc intracytoplasmic inclusion body[8].

It is noted that confirmatory detection of rotaviral infection of diarrheic dead calves is very important but application of different confirmatory diagnostic tests like ELISA and RT-PCR are expensive. In this view, the objective of the study was selected to identify of microscopic lesions of rotaviral infected dead calves through postmortem examination and similarly confirmed by ELISA and RT-PCR.

\section{Methodology}

\section{Sample Collection}

A total of 7 fecal samples/intestinal contents and tissue samples were collected from dead cattle calves of up to 45 days of age by postmortem examinations having history of clinical diarrhea at the time of mortality from the selected areas during the period July 2015 to June 2016. Fecal samples/intestinal contents were collected from intestine using a disposable latex glove. All standard precautionary measures were taken to avoid contamination of samples and transported to the clinical pathology laboratory on ice and stored at -20 until processing. Intestinal tissue samples showing lesions suggestive of rotavirus infection were collected and preserved in Bouin's solution for histopathological examinations.

\section{Sample Processing}

A 10\% fecal suspension of individual sample was prepared in phosphate buffered saline (pH 7.2) and clarified by centrifugation at $13,000 \mathrm{rpm}$ at $4^{\circ} \mathrm{C}$ for 10 minutes. The supernatant was separated and allocated into two aliquots. After processing, one aliquot was used to detect bovine rotavirus applying the direct sandwich ELISA and rest aliquot stored at $-70^{\circ}$ Cuntil RNA extraction for RT-PCR. Intestinal tissue samples were also preserved in $10 \%$ buffered formalin until histopathological examinations.

\section{Histopathological Examination}

The collected tissue samples were examined for histopathological investigation following proper techniques. In brief, the samples were preserved in Bouin's solution for 2-3 days and subsequently, samples were made smaller size $(5 \mathrm{~mm}$ thickness) and washed over night in running tape water. Then the tissues were dehydrated by ascending ethanol series to prevent shrinkage of cells as per following schedule. The tissues were dehydrated in 50\%, 70\%, 70\%, 80\%, 90\%, 95\%, 100\%, 100\%, $100 \%$ alcohol, one hour in each; one hour in absolute alcohol and absolute xyline mixture; two hours in $100 \%$ xyline, impregnation was done in melted paraffin $\left(60^{\circ} \mathrm{C}\right)$ for 3 changes and two hours for each change. After this step, sample was kept at room temperature overnight for drying. Then a block of sample was made using melted paraffin. These blocks of tissue sample were dried at room temperature. Then the blocks were sectioned with a microtome at 5 - $\mu \mathrm{m}$ thickness. A small amount of citric acid (or gelatin) was added to the water bath $\left(60^{\circ} \mathrm{C}\right)$ for better adhesion of the section to the slide. The tissue sections were allowed to spread on warm water both at $60^{\circ} \mathrm{C}$. Then the sections were taken on grease free clear slides. The slides containing section were dried room temperature and kept in cool place. Finally, the slide was prepared for routine hematoxylin and eosin staining.

The sectioned tissues were deparaffinized in two changes of xyline (two minutes in each). Then the sectioned tissues were rehydrated through descending grades of alcohol $(100 \%, 100 \%$, $95 \%, 80 \%$ and $70 \%$; two minutes in each) followed by washing in running rape water for five minutes. Then tissues were stained with Harries hematoxylin for ten minutes and then washed in running tap water for 15 minutes. Then the tissues were differentiated in $1 \%$ acid alcohol (1part HCL and 99 parts $70 \%$ alcohol) by 2-4 quick dips. Again, the slide washed in running tap water for 5 minutes and followed by 2-4 dips in ammonium. Again, the tissue sample was washed in running tap water for 10 minutes. After washing, the sections were stained with $1 \%$ eosin for two minutes. Again, the tissue was dehydrated in ascending grades of alcohol $(70 \%, 80 \%, 95 \%, 100 \%$ and $100 \%$ alcohol; 30 sec, $45 \mathrm{sec}, 2 \mathrm{~min}, 2 \mathrm{~min}$ and $2 \mathrm{~min}$ in each step; respectively). Then the tissue sample was treated in absolute alcohol and xyline mixture, $100 \%$ xyline for 2 minutes in each step. Finally, the slide was kept in $100 \%$ xyline until mounting. Then stained slide were mounted with cover slip using DPX mountant. Then slides were dried at room temperature and examined under light microscope at low (10x) and high (40x, 100x) power.

\section{ELISA for Detection of Rotavirus}

All 7 fecal samples were screened for the presence of bovine rotavirus antigen using sandwich ELISA kit (Bio-X Diagnostics, Belgium) as per manufacturer's recommended protocol.

\section{RNA Extraction}

All the 7 fecal samples were used for viral RNA extraction. Rotavirus RNA was extracted from the fecal suspension using QIAamp Viral RNA mini kit (Qiagen/Westburg, Leusden, Netherlands) following the manufacturer's instructions. The extracted RNA was stored at $-80^{\circ} \mathrm{C}$.

\section{RT-PCR}

The RT-PCR reaction was performed by using a QIAGEN One Step RT-PCR Kit (QIAGEN) for the confirmation of bovine rotavirus A. The primers used for amplifying a 294-bp fragment of the VP6 gene of rotavirus A, the sequence of the upstream primer was 5'-ACCACCAAATATGACACCAGC-3'; the sequence of the downstream primer was 5'-CATGCTTCTAATGGAAGC-3'[9]. Before applying RT-PCR reaction, $7 \mu$ l of viral dsRNA were denatured at $95^{\circ} \mathrm{C}$ for 5 minutes and chilled immediately for 5 minutes. Then, reaction was carried out under the following conditions: reverse transcription at $50{ }^{\circ} \mathrm{C}$ for 30 minutes, then PCR with initial activation at $95{ }^{\circ} \mathrm{C}$ for 15 minutes followed by 
40 cycles of amplification $94{ }^{\circ} \mathrm{C}$ for 30 seconds, annealing at 55 ${ }^{\circ} \mathrm{C}$ for 1 minute and extension at $72{ }^{\circ} \mathrm{C}$ for 1 minute, and a final extension of $72{ }^{\circ} \mathrm{C}$ for 7 minutes. The amplified PCR products were subjected to electrophoresis on $1.5 \%$ agarose gel stained with ethidium bromide and observed under ultraviolet light. Specific amplicon size for VP6 was observed on the stained gel.

\section{Results}

\section{Detection of Bovine Rotavirus by ELISA and RT-PCR}

Out of $7 \mathrm{fecal} /$ intestinal samples from diarrheic calves screened by ELISA (Figure 1) and, RT-PCR (Figure 2), same 4 samples were also positive for bovine group A rotavirus by ELISA and RT-PCR.
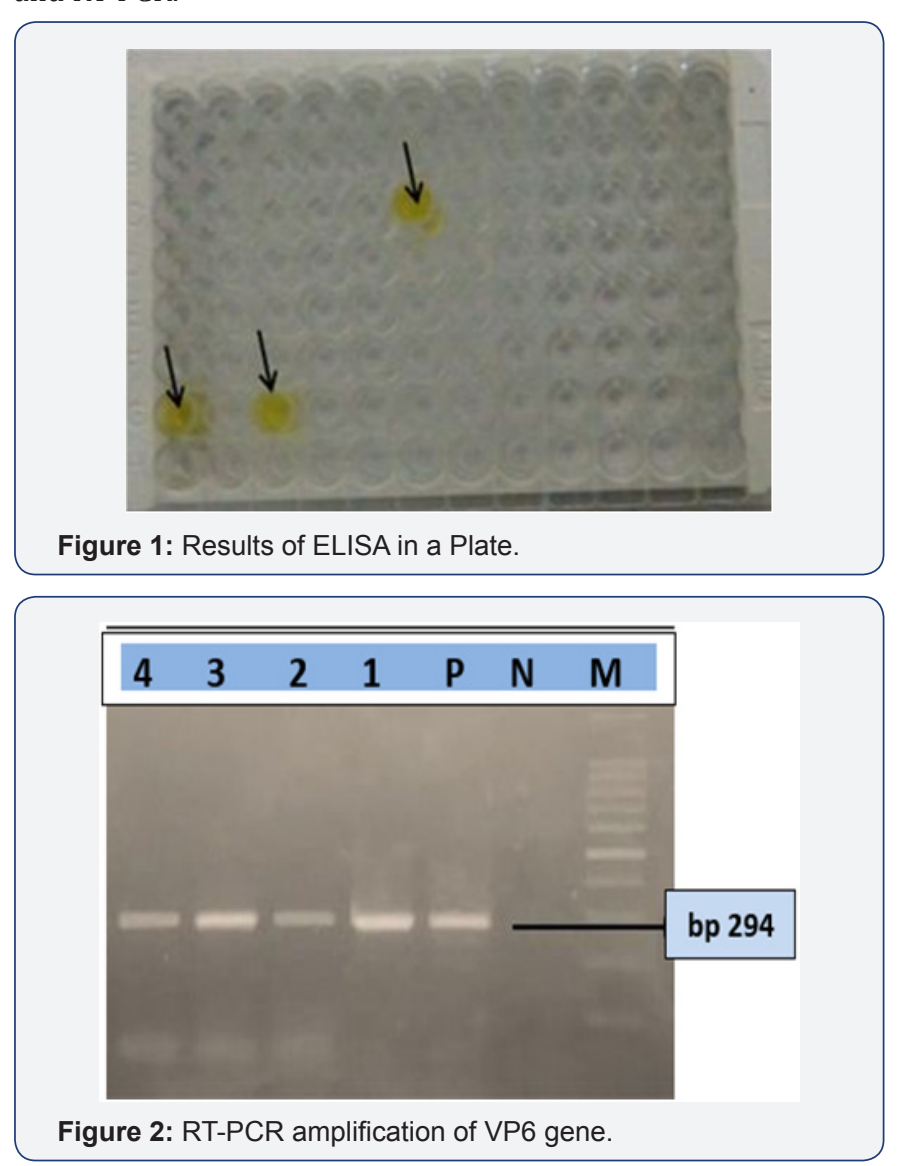

\section{Gross Lesion}

There were no gross pathological lesions revealed except mild catarrhal changes in the small intestine with congestion of both mucosa and serosal layers.

\section{Histopathological Changes}

All the seven samples were studied for histipathological changes for bovine rotavirus infection. Out of 7 samples, 4 samples showed microscopic changes related rotaviral infection. Blunting of villi and edema in lamina propria were found microscopically (Figures 3-5). Severe infiltration of lymphocytes was seen in mucosa occasionally extending up to the sub mucosa (Figure 5). Eosinophilic intracytoplasmic inclusion bodies were seen in the mucosal epithelial cells and a nuclear fragmentation was observed in few epithelial cells (Figures 6-8). The changes were seen in the mucosa of the duodenum, jejunum and ileum which revealed engorged capillaries and infiltration of lymphocytes. Jejunum showed engorged blood vessels in mucosa, sub mucosa and serosa with lymphocytic infiltration in mucosal layer. Desquamated epithelial cells were observed in the lumen. In ileum the changes were characterized by desquamation and necrosis of crypts with villous atrophy. Infiltration of lymphocytes could be seen in inter villous areas. In Peyer's patches, the lymphocytes were thinly populated, clumped, and few areas showed nuclear fragmentation and apoptotic bodies.
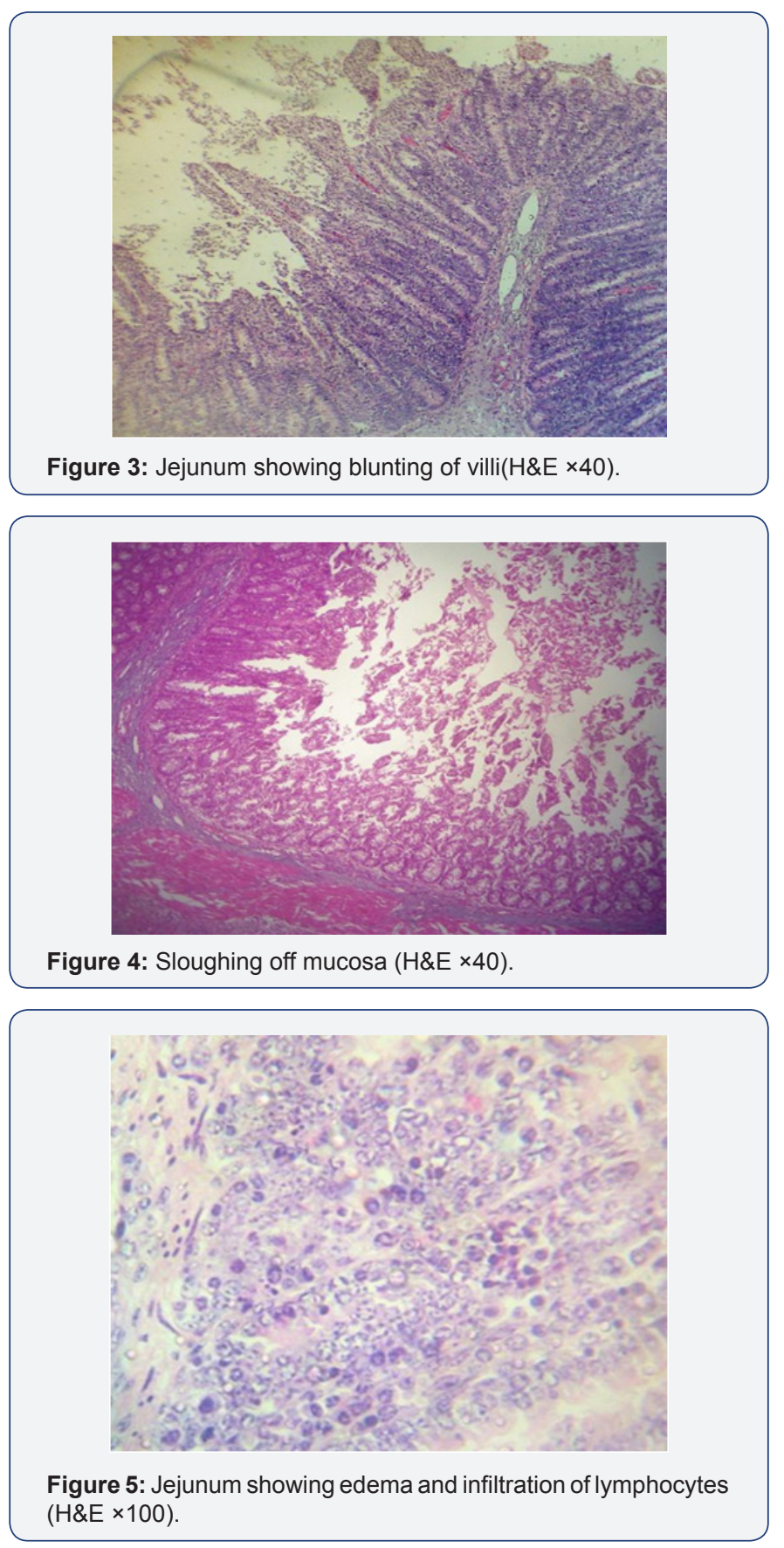

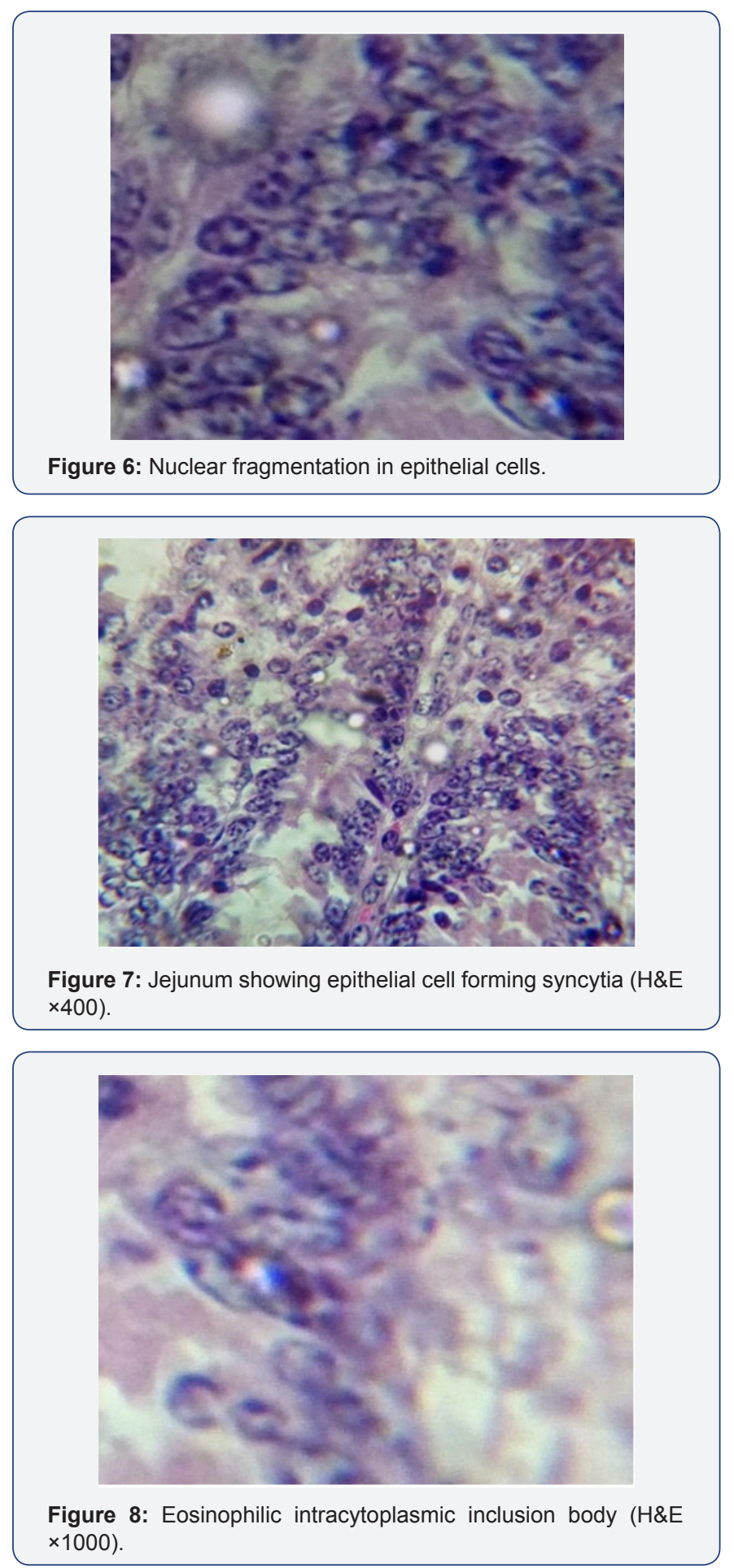

\section{Discussion}

In the present study seven calves died with the history of diarrhea revealed mild catarrhal changes in the small intestine with congestion of both mucosa and serosal layers and these changes were in agreement with earlier report. ELISA and RTPCR screening of the fecal samples/ intestinal contents for the presence of rotavirus antigen revealed positivity in 4 calves thus confirming rotavirus infection. The presence of intracytoplasmic inclusion bodies in small intestinal enterocytes was indicative of viral etiology. The characteristic changes as blunting of villi, thickening of crypts etc., was observed in the present study were suggestive of rotaviral etiology. The thinning of mesenteric lymph nodes was suggestive of lymphocytic migration as seen in the form of infiltration in mucosa. Previous studies confirm that rotaviruses are not able to produce severe gross changes in intestinal mucosa by their own [10]. The histological changes in ileum characterized by desquamation and necrosis of crypts with villous atrophy, infiltration of lymphocytes in inter villous areas and decreased population of lymphocytes in Peyer's patches and mesenteric lymph node were in agreesment with earlier reports $[1,6,11]$.

Rotavirus infection is implicated mainly in neonatal calves. The gross lesions of rotavirus infection are the nonspecific findings of undifferentiated neonatal diarrhea in calves. However, histopathology could be indicative; blunt, club-shaped villi, mild or moderate villus atrophy and perhaps villus fusion may be present $[12,13]$. Villi covered by low columnar, cuboidal, or flattened surface epithelium with a poorly defined brush border. There is usually a moderate proprial infiltrate of mononuclear cells and eosinophilic or neutrophils and hypertrophic crypts may be evident. Lesion and viral antigen always should be sought in the distal small intestine and preferably several sites along its length $[13,14]$.

Rotaviruses replicate in the non-dividing mature enterocytes near the tips of the villi, suggesting that differentiated enterocytes express factors required for efficient infection and replication [15]. The severity and localization of rotavirus intestinal infection vary among animal species and between studies; however, the pathological changes are almost exclusively limited to the small intestine. In various animal models, rotavirus infection was associated with virtually no visible microscopic lesions; slight lesions, such as enterocyte vacuolization and loss; or larger changes, such as villus blunting and crypt hyperplasia. Inflammation is generally mild compared to that for other intestinal pathogens. This picture of pathology suggests that there is no absolute correlation between histological lesions and disease symptoms. Rotavirus infection alters the function of the small intestinal epithelium, resulting in diarrhea. The diarrhea was generally considered to be malabsorptive, secondary to enterocyte destruction [4].

The mucosa of small intestine of the neonatal calf consists of long finger-like villi and pathological changes due to different infectious agents are almost similar [12]. The most significant pathological lesion consists of stunting and thickening of the villi and the villi are fused leading to a flat mucosa in the most severe cases. The epithelium covering such villi is generally cuboidal and in rotavirus infections squamous epithelium covering the tip of some villi may be seen $[12,16]$. Erosion of small areas of epithelium has been observed in rotavirus infections. 
Rotavirus, by itself, causes slight to moderate shortening (atrophy) of villi in the jejunum and ileum. The villi are blunt and covered by immature epithelial cells. When the rotavirus is accompanied by a pathogenic strain of Escherichia coli, the lesions are similar but more severe. There is degeneration and sloughing of cells from the villous tips, and bacteria are adherent to cell surface. Stunting and fusion of villi, exfoliation, disarrangement and vacuolation of enterocytes and the presence of cuboidal enterocytes were observed in infected calves but not in rotavirus-free control calves $[17,18]$. Lesions predominated in the upper small intestine, where rotavirus was most abundant, especially on the first two days of virus excretion. The numbers of enterocytes infected with rotavirus diminished before the lesions resolved.

In our study, histopathological changes agreed with the positivity of ELISA and RT-PCR test results. Microscopic lesions like, edema, infiltration of lymphocytes, nuclear fragmentation, syncytia formation, eosinophilic intracytoplasmic inclusion body observed only in those specimens that were the rotavirus cases confirmed by ELISA and RT-PCR.

\section{Conclusion}

Rotavirus infection in the present study revealed rotavirus specific lesions, such as denudation at tips of the villi, villus blunting and necrosis of crypts along with intracytoplamic inclusion bodies. The presence of rotavirus antigen in clinical samples/affected tissues was also confirmed by ELISA and RTPCR.

\section{References}

1. Dhama K, Chauhan R, Mahendran M, Malik S (2009) Rotavirus diarrhea in bovines and other domestic animals. Veterinary research communications 33(1): 1-23.

2. Chauhan R, Singh N (1996) Epidemiology of rotavirus infection in calves in India. International Journal of Animal Sciences 11(1): 221223.

3. Kim HJ, Park JG, Matthijnssens J, Lee JH, Bae YC, et al. (2011) Intestinal and extra-intestinal pathogenicity of a bovine reassortant rotavirus in calves and piglets. Veterinary microbiology 152(3-4): 291-303.
4. Blanchard PC (2012) Diagnostics of dairy and beef cattle diarrhea. Veterinary Clinics: Food Animal Practice 28(3): 443-464.

5. Luna LG (1968) Manual of histologic staining methods of the Armed Forces Institute of Pathology.

6. Suresh T, Rai R, Dhama K, Rai S, Sawant P, et al. (2011) Pathology of rotavirus infection in calves and detection of viral antigen by ELISA and FAT. Indian Journal of Veterinary Pathology 35(1): 1-3.

7. Guerrero CA, Santana AY, Acosta O (2010) Mouse intestinal villi as a model system for studies of rotavirus infection. Journal of virological methods 168(1-2): 22-30.

8. Suresh T, Rai R, Wani M, Damodaran T, Dhama K (2013) Detection of bovine rotavirus in neonatal calf diarrhoea by elisa, fat and transmission electron microscopy. International Journal of Current Research 5: 1935-1939.

9. Maes RK, Grooms DL, Wise AG, Han C, Ciesicki V, et al. (2003) Evaluation of a human group a rotavirus assay for on-site detection of bovine rotavirus. Journal of clinical microbiology 41(1): 290-294.

10. Chauhan R, Dhama K, Mahendran M (2008) Pathobiology of rotaviral diarrhea in calves and its diagnosis and control: A review. Journal of Immunology and Immunopathology 10: 1-13.

11. Chauhan R, Singh N (1992) Detection of rotavirus infection in calves employing immunofluorescence. Journal of Applied Animal Research 1(1): 51-55.

12. Foster D, Smith GW (2009) Pathophysiology of diarrhea in calves. Veterinary Clinics: Food Animal Practice 25(1): 13-36.

13. Cho Yi, Yoon KJ (2014) An overview of calf diarrhea-infectious etiology, diagnosis, and intervention. Journal of veterinary science 15(1): 1-17.

14. Jubb KVF (1985) Pathology of domestic animals 3E. Academic press, USA.

15. Ramig RF (2004) Pathogenesis of intestinal and systemic rotavirus infection. Journal of virology 78(19): 10213-10220.

16. Lorenz I, Fagan J, More SJ (2011) Calf health from birth to weaning. II. Management of diarrhoea in pre-weaned calves. Irish veterinary journal 64: 9.

17. Ciarlet M, Conner ME, Finegold MJ, Estes MK (2002) Group A rotavirus infection and age-dependent diarrheal disease in rats: a new animal model to study the pathophysiology of rotavirus infection. Journal of virology 76(1): 41-57.

18. Abe M, Ito N, Morikawa S, Takasu M, Murase T, et al. (2009) Molecular epidemiology of rotaviruses among healthy calves in Japan: isolation of a novel bovine rotavirus bearing new $\mathrm{P}$ and $\mathrm{G}$ genotypes. Virus research 144(1-2): 250-257.

\section{Your next submission with Juniper Publishers} will reach you the below assets

- Quality Editorial service

- Swift Peer Review

- Reprints availability

- E-prints Service

- Manuscript Podcast for convenient understanding

- Global attainment for your research

- Manuscript accessibility in different formats

( Pdf, E-pub, Full Text, Audio)

- Unceasing customer service

Track the below URL for one-step submission https://juniperpublishers.com/online-submission.php 